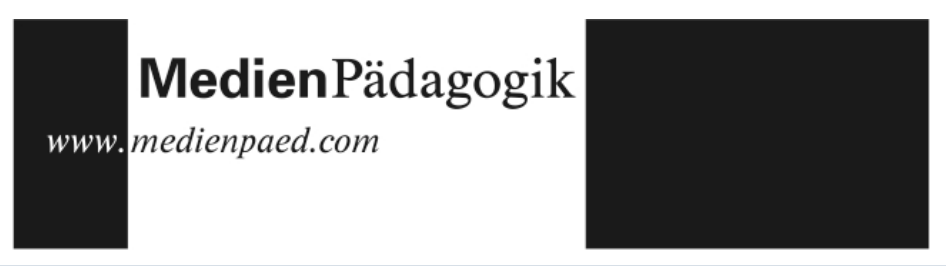

Rezensionen

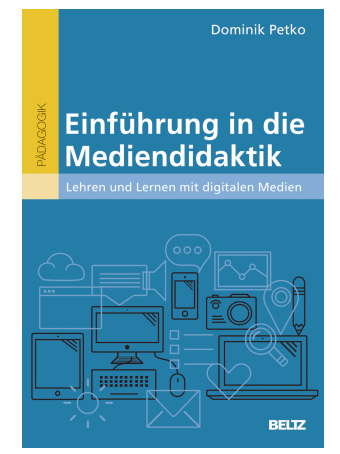

Dominik Petko

Einführung in die Mediendidaktik: Lehren und Lernen mit digitalen Medien

1. Auflage, Herausgegeben von Eiko Jürgens

Weinheim und Basel: Beltz, 2014. 184 Seiten

ISBN: 978-3-40725678-2

$€ 24.95$; CHF 35.50

\title{
Mediendidaktik zwischen Wissenschaft und Praxis
}

Es mag eine Angewohnheit sein, wissenschaftliche Fachbücher zunächst nach Inhalts- und Literaturverzeichnis sowie nach der Einleitung und nach dem Fazit zu beurteilen. Gerade diese Bestandteile erlauben essenzielle Einblicke in den Charakter eines Buches sowie in Standpunkte und disziplinäre Prägung(en). Aus diesem Grund wurde auch die Einführung in die Mediendidaktik von Dominik Petko, Professor für Medienpädagogik und empirische Methoden an der Pädagogischen Hochschule Schwyz, zunächst auf diese Weise geprüft. So wirkten die Inhalte auf den ersten Blick «klassisch», das Literaturverzeichnis weist eine Reihe bekannter (inter-)nationaler Publikationen zur Mediendidaktik auf und in Einleitung und Schluss fallen zwei Aspekte auf, die besonders herausgestellt werden. Dazu gehört der konsequente Fokus auf die digitalen Medien, der sich durch das gesamte Buch zieht, sowie die herausragende Bedeutung der Lehrperson im Spannungsfeld unterschiedlicher (Umwelt-)Einflüsse, die im sechsten Kapitel als «Rahmenbedingungen von Schule» herausgearbeitet werden.

Während letztere Rahmung angesichts der primären Zielgruppe des Buchs - bereits aktive und angehende Lehrpersonen - erwartbar war, ist Ersteres angesichts fortschreitender Mediatisierung überraschend, aber doch nah an den Herausforderungen der Praxis. Mehr noch: Diese Fokussierung zeigt die rasante Geschwindigkeit, mit der das für den Unterricht nutzbare Medienangebot über Buch, TV/Video und Radio hinaus wächst, sowie die Notwendigkeit, als Lehrperson, aber auch als Institution Schule immer wieder auf augenscheinlich «neue» Medien zu reagieren, im besten Fall proaktiv und auf Basis fundierter didaktischer Konzepte (nicht zugunsten technologischer Hypes). 
Entsprechend werden auch die Ziele des Buchs formuliert: Der Autor will «eine praxisorientierte Einführung in die Möglichkeiten und Herausforderungen digitaler Medien in Schulen geben und damit auch zum Nachdenken über die Rolle von Lehrpersonen in der Informationsgesellschaft anregen» (S. 10). Weiter heisst es: «Damit das vorliegende Lehrbuch nicht übermorgen schon wieder veraltet ist, geht es nicht nur von aktuellen Beispielen aus, sondern vermittelt ein eher konzeptionelles Wissen» (S. 11). Beide Fokussierungen sind wichtig, wenn nicht gar erforderlich, um eine Einführung in die Mediendidaktik unter den Vorzeichen der Mediatisierung anzubieten.

Blickt man von der Oberfläche weiter in die Inhalte des Buchs, finden sich nach der kurzen Einleitung Ausführungen zum Medienbegriff und zum Lernen «an sich» - Zusammenhänge, die vielfach in mediendidaktischen Publikationen zu finden sind und in komprimierenden Einführungen stets eine Berechtigung haben. Es folgt ein Kapitel zu Lern- und Unterrichtsmedien, das nicht nur verschiedene digitale Medienangebote exemplarisch aufgreift, sondern mithilfe von Ankern aus Theorie, Empirie und Praxis Perspektiven des Medieneinsatzes in der Schule deutlich macht. Die folgende fachdidaktische Rahmung ist hilfreich, da Mediendidaktik in der Sichtweise von (angehenden Lehrpersonen) oft als Teil von Fächern (bspw. Deutsch, Mathematik) wahrgenommen wird. Einleitung und Schluss runden das Buch mit gesellschaftlichem Bezug ab, denn: «Die digitale Revolution in den Schulen hat gerade erst begonnen» (S. 159).

\section{(Medien-)Didaktische Konzeption über Hypes und Technologie hinaus}

Die Einführung in die Mediendidaktik erweist sich insgesamt als kurzweiliges Buch. Dies liegt nicht zuletzt an der Länge (knapp 160 Seiten), insbesondere aber auch am internen Aufbau, der gewählten Detailliertheit sowie der Anschaulichkeit durch aktuelle Themen, Beispiele, Links etc. Während das Inhaltsverzeichnis an vielen Stellen noch eine klassische Konturierung erwarten liess (vgl. oben) und dort integrierte, rhetorische Fragen erst ungewöhnlich erschienen, erweist sich der Aufbau nach der Gesamtlektüre als geglückt. Inhaltlich werden unterschiedliche Granulierungsgrade angeboten. Sie sind dabei weitestgehend ausgewogen. Lediglich Computerspiele/Serious Games werden überproportional tief besprochen, was offenbar dem persönlichen Interessenfeld des Autors sowie dem Kontext Schule/Unterricht zuzuschreiben ist. Zugleich wird stets versucht, ausgehend von (schul-)praktischen Fragen Brücken zur mediendidaktischen Theorie und zugehöriger Empirie zu schlagen. Letzteres ist erwähnenswert, da der An- 
wendungsbezug empirischer Befunde für Lehrpersonen vielfach ausbleibt und vorliegende Ergebnisse von der Zielgruppe des Buchs wenig rezipiert, geschweige denn in passende didaktische Konzepte gewendet werden (können). Das Buch macht hingegen an mehreren Stellen klar, wo und an welchen Stellen solche Ergebnisse für die Unterrichtspraxis bedeutsam sind, ohne empirische Befunde als Grundlage von Unterricht und Konzeption zu sehr zu strapazieren. Hier ist schliesslich die Position des Autors unverkennbar, der sowohl praktischen (Gestaltungs-)Fragen auf unterschiedlichen Gestaltungsebenen nachgeht als auch der empirischen Untersuchung von Unterricht und Schule Bedeutung beimisst.

Die gewählte Position innerhalb der Mediendidaktik (Lehren und Lernen mit Medien) ist angesichts der propagierten Ziele und der Zielgruppe des Buchs verständlich. Sie macht - einmal mehr - eine engere Sicht auf Mediendidaktik auf, die sich innerhalb der Medienpädagogik neben der Medienerziehung verortet. Zwischen den Zeilen werden allerdings aktuelle Diskurse um die Verortung der Mediendidaktik als Fach sowie ihren möglichen normativen Charakter deutlich. Ob und inwieweit bereits im Schuldienst tätige oder angehende Lehrpersonen diese eher impliziten Hinweise und Stellungsnahmen aufgreifen oder nachvollziehen können, bleibt allerdings nach der Lektüre offen. So muss wohl im Rahmen von Lehrveranstaltungen oder Weiterbildungen mit Dozierenden geklärt werden, welche Fähigkeiten zur Umsetzung der beschriebenen mediendidaktischen Arrangements vonnöten sind, welche Förderperspektiven sich für den Einsatz von Medien in Unterricht und Schule ergeben, welche Rollen Akteure, Institutionen und Strukturen innerhalb des skizzierten Gefüges haben und welche Wechselwirkungen sich zwischen innen ergeben.

Dass im Buch argumentativ oft eine infrastrukturelle bzw. werkzeugorientierte Sicht auf den Medieneinsatz eingenommen wird, erfordert eventuell die gegenwärtige, nach wie vor schlechte Ausstattungslage der Schulen. Es könnte auch sein, dass Hard- und Software, die einer Integration in den Schulalltag bedürfen, für die Zielgruppe einen nachvollziehbareren Zugang zur Mediendidaktik bieten als konzeptionelle Fragen von Beginn an. Über diese Brücke «hinter» dem Medieneinsatz muss die Zielgruppe allerdings auch nach der Lektüre selbst gehen, was angesichts weit verbreiteter Haltungen und der vor allem in praktischen Handreichungen vorherrschenden Verknappung auf den technischen Gebrauch von (digitalen) Medien herausfordernd sein dürfte. 
Die vorliegende Einführung in die Mediendidaktik von Dominik Petko ist insofern ein knappes, aber in der Gesamtschau hilfreiches Werk, das der primären Zielgruppe einen Zugang zu vielfältigen Diskussionen und Fragestellungen im Zusammenhang mit digitalen Medien in Unterricht und Schule anbietet. Bei aller Übertragungsleistung zwischen den Kontexten dürfte das Buch auch für eine sekundäre Zielgruppe aus Hochschuldozierenden oder medienpädagogisch/-didaktisch tätigem Personal einige Hinweise für die Konzeption mediengestützter Lernumgebungen bieten. Dem Charakter einer Einführung wird es vor allem durch die gewählten Beispiele und durch den konsequenten Anwendungsbezug zu Lehr-Lernszenarien gerecht, ohne sich dabei in trendbezogener Aktualität zu verlieren. Hinsichtlich Theorie und Empirie müssen sich Lesende individuell vertiefen; Verknüpfungen zu relevanter Forschungsliteratur bestehen jedenfalls reichlich und sorgen hoffentlich dafür, dass bei der Zielgruppe Problembewusstsein für mediendidaktische Fragen erzeugt wird. Dazu gehört dann auch, sich neben diesem Lehrbuch weitere Einführungen zu erarbeiten, die der Autor eingangs (S. 10) selbst empfiehlt und die das Spektrum der (didaktischen) Positionen und Diskussionen letztlich erst komplettieren. 